\title{
BMJ Open Antibiotic-Loaded Bone Cement in Prevention of Periprosthetic Joint Infections in Primary Total Knee Arthroplasty: A Register-based Multicentre Randomised Controlled Non-inferiority Trial (ALBA trial)
}

Tesfaye H Leta (10 , 1,2 Jan-Erik Gjertsen,, ${ }^{2,3}$ Håvard Dale, ${ }^{2,3}$ Geir Hallan, ${ }^{2,3}$ Stein Håkon Låstad Lygre, ${ }^{2,4}$ Anne Marie Fenstad, ${ }^{2}$ Gro Sævik Dyrhovden, ${ }^{3}$ Marianne Westberg, ${ }^{5}$ Tina Stromdal Wik, ${ }^{6,7}$ Rune Bruhn Jakobsen,, 8 Arild Aamodt, ${ }^{10}$ Stephan Maximillian Röhrl, ${ }^{5}$ Øystein Johannes Gøthesen, ${ }^{3,11}$ Einar Lindalen, ${ }^{10}$ Stig Heir, ${ }^{12}$ Jarle Ludvigsen, ${ }^{13}$ Trond Bruun, ${ }^{14}$ Ann Kristin Hansen, ${ }^{15,16}$ Knut Erik Moen Aune, ${ }^{17}$ Marianne Warholm, ${ }^{18}$ John Petter Skjetne, ${ }^{19}$ Mona Badawy, ${ }^{20}$ Pål Høvding, ${ }^{2}$ Otto Schnell Husby, ${ }^{6}$ Øystein Espeland Karlsen, ${ }^{21}$ Ove Furnes ${ }^{2,3}$

To cite: Leta TH, Gjertsen J-E, Dale $\mathrm{H}$, et al. AntibioticLoaded Bone Cement in Prevention of Periprosthetic Joint Infections in Primary Total Knee Arthroplasty: A Registerbased Multicentre Randomised Controlled Non-inferiority Trial (ALBA trial). BMJ Open 2021;11:e041096. doi:10.1136/ bmjopen-2020-041096

- Prepublication history and additional material for this paper are available online. To view these files, please visit the journal online (http://dx.doi. org/10.1136/bmjopen-2020041096).

Received 29 May 2020

Revised 16 December 2020

Accepted 28 December 2020

\section{Check for updates}

(c) Author(s) (or their employer(s)) 2021. Re-use permitted under CC BY-NC. No commercial re-use. See rights and permissions. Published by BMJ.

For numbered affiliations see end of article.

\section{Correspondence to}

Dr Tesfaye H Leta;

tesfaye.hordofa.leta@helsebergen.no

\section{ABSTRACT}

Introduction The current evidence on the efficacy of antibiotic-loaded bone cement (ALBC) in reducing the risk of periprosthetic joint infections (PJI) after primary joint reconstruction is insufficient. In several European countries, the use of $A L B C$ is routine practice unlike in the USA where ALBC use is not approved in low-risk patients. Therefore, we designed a double-blinded pragmatic multicentre register-based randomised controlled noninferiority trial to investigate the effects of ALBC compared with plain bone cement in primary total knee arthroplasty (TKA).

Methods and analysis A minimum of 9,172 patients undergoing full-cemented primary TKA will be recruited and equally randomised into the ALBC group and the plain bone cement group. This trial will be conducted in Norwegian hospitals that routinely perform cemented primary TKA. The primary outcome will be risk of revision surgery due to PJI at 1-year of follow-up. Secondary outcomes will be: risk of revision due to any reason including aseptic loosening at 1, 6, 10 and 20 years of follow-up; patient-related outcome measures like function, pain, satisfaction and health-related quality of life at 1, 6 and 10 years of follow-up; risk of changes in the microbial pattern and resistance profiles of organisms cultured in subsequent revisions at $1,6,10$ and 20 years of followup; cost-effectiveness of routine ALBC versus plain bone cement use in primary TKA. We will use 1:1 randomisation with random permuted blocks and stratify by participating hospitals to randomise patients to receive ALBC or plain bone cement. Inclusion, randomisation and follow-up will be through the Norwegian Arthroplasty Register.

Ethics and dissemination The trial was approved by the Western Norway Regional Committees on Medical and
Strengths and limitations of this study

- To our knowledge, this is the first large pragmatic multicentre register-based randomised controlled non-inferiority trial designed to investigate the effects of routine antibiotic-loaded bone cement use in primary total knee arthroplasty in preventing subsequent revision due to periprosthetic joint infections.

- Register-based randomised controlled trial (R-RCT) represents new possibilities, pairing the power of randomisation with the simplicity of a quality register to detect clinically important differences in patient outcomes.

- R-RCT will facilitate large pragmatic interventional trials with adequate statistical power and low cost in the real-world setting.

- In this trial, the work load on the patient and the surgeon is minimal, and all follow-up is done electronically via web-based solutions through the Norwegian Arthroplasty Register, a well-established register, with high national coverage and good response rate.

- The limitation of this trial is that the surgeons are not blinded, which would yield bias, however, we believe the primary endpoint of this trial is not likely to be influenced by the surgeon knowledge of the cement used in the index surgery.

Health Research Ethics (reference number: 2019/751/ REK vest) on 21 June 2019. The findings of this trial will be disseminated through peer-reviewed publications and conference presentations.

Trial registration number NCT04135170. 


\section{INTRODUCTION}

Total knee arthroplasty (TKA) is an effective treatment for degenerative knee joint disease. ${ }^{1-3}$ The incidence of TKA is increasing worldwide ${ }^{4}$ and projected to reach 5 million by $2030 .{ }^{5}$ Despite the numerous perceived improvements in perioperative antimicrobial procedures, periprosthetic joint infection (PJI) following TKA remains a serious complication for patients and a burden for the healthcare system. ${ }^{67} \mathrm{PJI}$ is one of the most frequent causes of TKA revision surgery accounting for more than $15 \%$ of all revisions. ${ }^{89}$

PJI may result in a long hospital stay, increased risk of readmission and poor patient outcomes including decreased function and diminished quality of life. In worst case, it may lead to amputation or even death. ${ }^{10} 11$ The cost burden due to PJI is almost twice as high as those without PJI and the cost per PJI-related admission being around US\$80,000 in the USA. ${ }^{11} 12$

To reduce the risk of PJI, antibiotic-loaded bone cement (ALBC) has been widely used over the last 40 years. ${ }^{13} 14$ Therefore, the use of ALBC is standard practice in many European countries today. ${ }^{61516}$ However, in the USA, the use of ALBC is only approved for revision of infected arthroplasty and ALBC is not licensed for prophylactic use in primary arthroplasty for low-risk patients. ${ }^{15}{ }^{17}$ In Australia, the use of ALBC in arthroplasty surgery depends on the preference of the surgeons and hospitals. ${ }^{18}$

The use of ALBC and its efficacy in reducing PJI are persistently debated in the literature. ${ }^{7} 1516$ 19-53 The supporters of routine ALBC-use in primary arthroplasty claim that it reduces the risk of PJI ${ }^{76} 353841434652$ and consequently reduces patient suffering and costs without noticeable side effects. However, the opponents claim that the antibiotic in ALBC weakens the mechanical properties of cement and thus, increase the risk of aseptic loosening, ${ }^{54}{ }^{55}$ systemic toxicity or allergic reaction, ${ }^{28} 32-3456$ bacterial resistance ${ }^{24-27}$ and consequently increases patient suffering and cost. ${ }^{1520535758}$ Up to $8.4 \%$ of antibiotics in ALBC are released within 6 hours after surgery, followed by a low-dose release over months, which may be below both the minimum bactericidal concentration and the minimal inhibitory concentration $(\mathrm{MIC})^{27} 28$ Prolonged exposure to antibiotic at a lower concentration than MIC could lead to the development of mutational resistance in bacteria or selection of resistant strains. ${ }^{59}$ Thus, the theoretical benefit of prophylactic ALBC in reducing the risk of PJI should be weighed against its potential adverse effects.

The conclusions from both observational and randomised controlled trial studies on ALBC are inconsistent. ${ }^{1617394042434560}$ Chiu et a ${ }^{60}$ reported a significant reduction in risk of PJI by use of ALBC in primary TKA in patients with diabetes mellitus (high-risk patients). A study from Spain ${ }^{39}$ reported that the use of erythromycin and colistin-loaded cement in TKA did not lead to a reduction in the incidence of infection. Namba $e t$ $a t^{42}$ even reported a higher rate of infection at 1 year in the group treated with ALBC compared with plain bone cement $(1.4 \%$ vs $0.7 \%)$. Qadir et $a l^{17}$ also reported that the use of ALBC did not prevent PJI after primary TKA, even in high-risk patients. Several meta-analyses and systemic reviews studies have concluded that the use of $A L B C$ versus plain bone cement did not decrease the rate of deep infections in TKA. ${ }^{363748-5052}$

In Norway, antibiotic resistance is a limited but emerging problem. Antibiotic stewardship is important to ensure future effectiveness of antibiotics. ${ }^{61}$ Thus, in 2015 , the Norwegian government set goals to reduce antibiotics use in humans by $30 \%$ by $2020 .{ }^{62} 63$

The current evidence on the effectiveness of ALBC in reducing the risk of PJI after arthroplasty is insufficient ${ }^{28}$ and previous studies on ALBC use have indeed called for large, prospective, and preferably multicentre studies to justify routine use of ABLC in primary arthroplasty. ${ }^{19} 20$ In Norway, the proportion of ALBC use in hybrid or fully cemented primary TKA increased from around $70 \%$ in 1994 to nearly $100 \%$ in $2019 .{ }^{64}$ Without a definitive trial, patients will be exposed to a treatment of uncertain efficacy that may drive antibiotic resistance at a higher immediate and future cost.

Register-based randomised controlled trial (R-RCT) represents new possibilities, pairing the power of randomisation with the simplicity of a quality register to detect clinically important differences in patient outcomes. ${ }^{65} 66$ R-RCT may facilitate large pragmatic interventional trials with adequate statistical power and low cost in the realworld setting. ${ }^{65}$

Therefore, we present a trial protocol for a large pragmatic multicentre register-based randomised controlled non-inferiority trial aiming to investigate the effects of ALBC compared with plain bone cement in primary TKA. We hypothesise that: (1) Plain bone cement is noninferior to ALBC in risk of revision due to PJI following primary TKA; (2) Patient reported outcome measures (PROMs) of patients operated with ALBC in primary TKA are similar compared with that of patients operated with plain bone cement; (3) Routine use of ALBC in primary TKAs does not result in a change in the microbial pattern and resistance profiles of organisms cultured in subsequent revision due to PJI and (4) Routine ALBC use is as cost-effective as plain bone cement use in primary TKA.

\section{MATERIALS AND ANALYSIS Study design}

This is a double blinded, multicentre, register-based randomised controlled non-inferiority trial. The study will include patients undergoing full-cemented primary TKA. The patients will be randomised to TKA with either ALBC or plain bone cement through the Norwegian Arthroplasty Register (NAR). Our trial protocol follows the Standard Protocol Items: Recommendations for Interventional Trials reporting guideline for clinical trials. ${ }^{67}$ A summary of the trial design is presented graphically in figure 1 . 


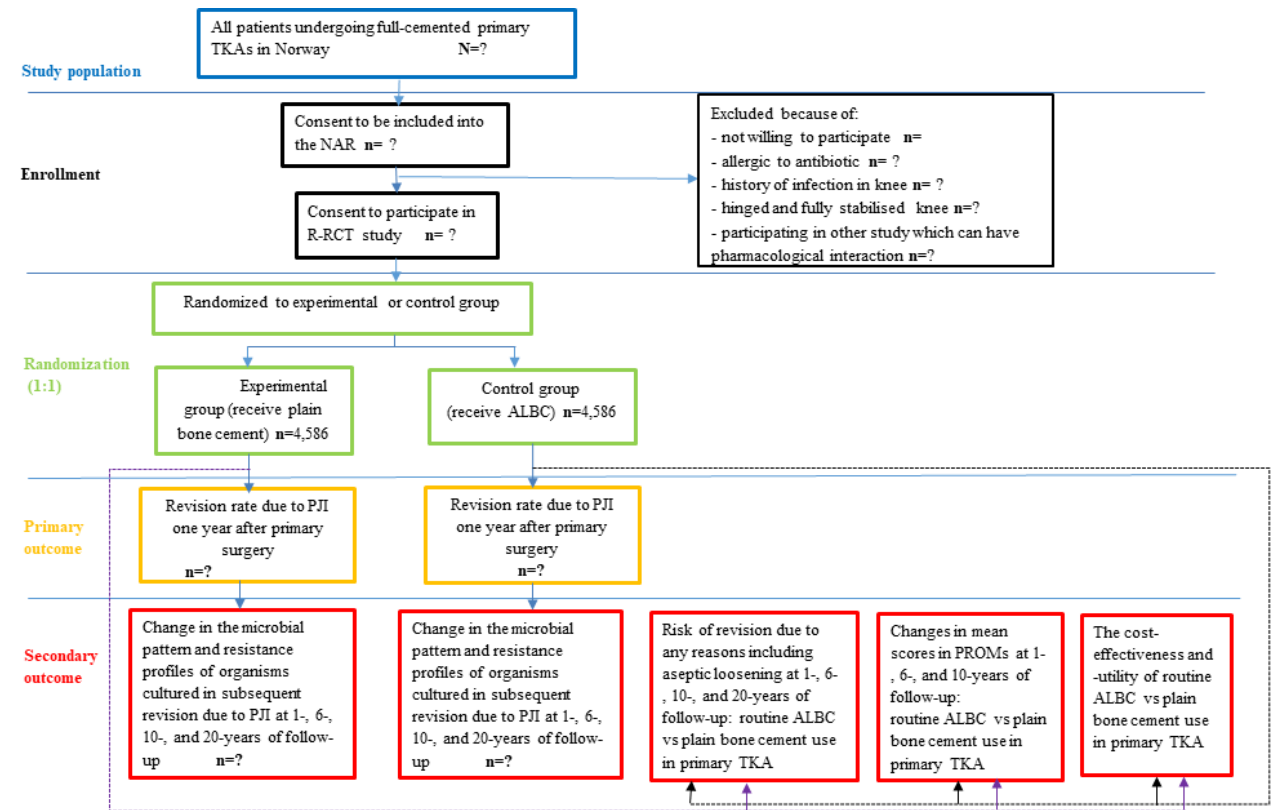

Figure 1 Flowchart (summary of trial design). ALBC, antibiotic-loaded bone cement; NAR, Norwegian Arthroplasty Register; $\mathrm{PJI}$, periprosthetic joint infection; R-RCT, register-based randomised controlled trial; TKA, total knee arthroplasty.

\section{Study setting and population}

All Norwegian hospitals that perform cemented TKAs routinely are invited to participate in this study. In Norway, nearly 6,000 TKA surgeries are performed annually and over $80 \%$ them are cemented. ${ }^{68}$ All patients undergoing full-cemented primary TKA are eligible for participation irrespectively of the diagnosis leading to TKA. The indications for TKA and routines during the patient's stay at the hospital will be as usual practice at each hospital. Exclusion criteria will be; any history of infection in the knee, a need for fully stabilised or hinged TKA, a history of allergy to the antibiotics used in the cement, inability or not willing to consent for inclusion in NAR or the trial, and participation in other studies that might have pharmacological interaction with this trial.

\section{Definitions}

Revision is defined as the removal, addition and/or exchange of part of an implant or the whole implant. 'Second revision' is defined as a subsequent revision. Since the NAR records revision surgery due to deep infection, PJI in ALBA trial means only surgically treated PJI and excludes superficial infections and infections treated only by antibiotics.

\section{Informed consent}

An orthopaedic surgeon or nurse will introduce the trial to the patients. Patients fulfilling the inclusion criteria will receive verbal and written information about the trial prior to surgery and will be asked to sign a consent form (online supplemental file 1 -informed consentNorwegian version). We will inform the patients that participation in the study is entirely voluntary and any decision they make will not influence their future healthcare. Participants will also be informed of their right to withdraw from the trial whenever they desire without any reason they need to supply for such decision, however, their data acquired prior to withdrawal will be maintained in the study database and included in the analysis to avoid bias.

\section{Randomisation and allocation concealment}

Both the randomisation and reporting will be web based, governed from the NAR. The randomisation will be done directly in a web-based registration system before the start of the surgery. We will use 1:1 randomisation with random permuted blocks (of 4, 6 or 8 ) and stratify by participating hospitals to randomise patients to receive ALBC or plain bone cement. ${ }^{6970}$ The data that will be collected is the core dataset in the NAR: patient identity, date of operation, indication for primary TKA, type of implant and other surgeryrelated factors. Information on patient-related factors like sex, age and comorbidities is also registered. The unique social security number of each Norwegian inhabitant links the primary TKA to any subsequent revisions or reoperation, and to the National Population Register, which provides information on death and emigration. Revision is defined as the removal, addition and/or exchange of part of an implant or the whole implant, whereas, reoperation is defined as surgery associated with the TKA without removing, adding and/or exchanging prosthesis parts. The NAR records both reoperations and revision surgery due to deep infection, but not conservatively/suppressive treated PJI. Hence, only surgically treated PJI will be included in this trial. The surgeon fills in the register form immediately after surgery. PROMs will be collected electronically per the NAR standard routine. There will be manual back-up solutions in the case of temporal technological problems with randomisation or reporting. 
Every hospital participating in this trial needs to have both types of cement (with and without antibiotics) available at all times. The catalogue number of cement used in each operation is reported by scanning the barcode on the package of the used cement. If the cement used does not match the cement drawn by randomisation, the registration will still be used to perform intention to treat-analyses and as treated-analyses. The surgical procedures are performed according to the protocols at each hospital.

In case of any revision, the surgeon will fills in a revision report, similar to the report after primary TKA. The surgeon will then report on the indication for revision surgery. In the case of a reported revision due to deep infection or aseptic loosening, additional information on biochemical and bacteriological findings will be collected to validate the diagnosis of PJI and to collect information on bacteriological findings and antimicrobial resistance.

\section{Blinding}

Confounding and selection bias that might affect observational studies will be minimised through randomisation; however, randomisation does not prevent subsequent biased assessment of outcomes. ${ }^{71}$ Patients and data analysts will, therefore, be blinded in this trial. Blinding of the surgeon will not be possible because the surgeon recognises the cement type and has to document the type of cement in the electronic registration form. We believe the primary endpoint of this trial is not likely to be influenced by the surgeon knowledge of the cement used in the index surgery. The data analyst will be blinded for the group allocation until the entire trial analysis has been completed to minimise the risk of bias that may be introduced during the statistical analysis because of the selective use and reporting of statistical tests. ${ }^{71}$

\section{Sample size and statistical power}

Earlier register study showed that ALBC and plain bone cement had $1 \%$ and $1.5 \%$ risk of revisions for deep infection 1 year after primary total hip arthroplasty, respectively. ${ }^{7}$ This trial is a non-inferiority trial ${ }^{72} 73$ assuming a 1-year revision rate of $1 \%$ following primary TKA with ALBC. To show the non-inferiority of plain bone cement to ALBC with respect to revision due to PJI 1 year after primary TKA, with at least $80 \%$ power, and a noninferiority margin of 0.15 percentage points at a onesided significance level of $0.025,9,172$ patients $(4,586$ in each group) would need to be enrolled.

Based up on the non-inferiority margin of 0.15 , the number needed to harm will be 667 (100 divided by $0.15)$. In other words, we assume that around 670 patients needed to undergo primary TKA surgery with plain bone cement to cause harm (revision due to PJI) in one patient that would not otherwise have been harmed.

\section{Interim analysis}

Interim analyses will be carried out after 1,000 and 6,000 patients have been enrolled. If we consider stopping the trial early, the O'Brien-Fleming stopping rule will be used to declare a statistically significant difference in the primary outcome between the two groups. ${ }^{74}$ The trial steering committee will based on the interim analyses make a recommendation to the Norwegian National Advisory Unit on Arthroplasty and Hip Fractures' Steering Committees, as to whether the trial should be stopped early or not. This recommendation will also take other sources of evidence such as secondary outcomes and safety data into consideration.

\section{Planned intervention}

The patients randomised to ALBC (control group) will receive bone cement with antibiotics and those randomised to plain bone cement (experimental group) will receive bone cement without antibiotics. The type (brands) of bone cement will be determined according to each hospital's standard protocol. All patients will receive systemic antibiotic prophylaxis according to the national guidelines. ${ }^{76}$ According to these guidelines, currently, patients should receive repeated doses of a first or second-generation cephalosporin or cloxacillin, or in the case of allergy, clindamycin; first dose should be administered $30-60$ min preoperatively ${ }^{76}$ Systemic antibiotic prophylaxis must constitute a maximum of four doses and be administered within the first 24 hours of surgery.

\section{Quality control}

All quality and safety aspects of this trial, including informed consent, inclusion and exclusion criteria, data quality as well as adverse events will be regularly monitored by the trial steering committee and by the Norwegian National Advisory Unit on Arthroplasty and Hip Fractures.

\section{Data collection and assessment points}

Patient recruitment and data collection will be expected to start in late 2020. Baseline and surgical-related information will be collected on the day of surgery whereas PROMs data collected preoperative and at 1, 6 and 10 years follow-up. Assessment will occur at predetermined endpoints. Assessment on primary outcome and reports or presentations of preliminary results will be after completion of inclusion and full 1-year follow-up. First assessment will be when included patients have got a full 1-year follow-up. Latter assessments will be at 6,10 and 20years follow-up. The PROMs questionnaires will consist of general information (ie, education, smoking status, height and weight and diabetic status), Visual Analogue Scales for pain and satisfaction, Knee injury and Osteoarthritis Outcome Score for functional outcome, the 5-level Euroqol for health-related quality of life (HRQoL), University of California, Los Angeles activity score and Charnley score. Microbial pattern and resistance profiles of organisms cultured in subsequent revision will be followed up to death or emigration. The cost-effectiveness of ALBC use vs plain bone cement use in primary TKA will also be assessed. 


\section{Data management, access and sharing}

All this trial study data will be stored in the NAR Oracle database. On receipt of the data, the NAR personnel, blinded to the group allocation, will make a visual check of the data and query all missing, implausible and inconsistent data. During the study, the investigators have access to the trial data and statistic support from the NAR. Data generated by this trial will be made available after completion of patient inclusion and full 1-year follow-up, and will be available on reasonable request. Data access request will be reviewed by the Trial Steering Committee and by the Norwegian National Advisory Unit on Arthroplasty and Hip Fractures. Requestors will be required to sign the NAR data access agreement.

\section{Outcomes}

Primary outcome will be revision due to PJI at 1-year follow-up. Secondary outcomes will be:

- Risk of revision due to any reason including aseptic loosening at 1, 6, 10 and 20 years of follow-up.

- PROMs like function, pain, satisfaction and HRQoL at 1,6 and 10 years of follow-up.

- Risk of changes in the microbial pattern and resistance profiles of organisms cultured in subsequent revisions at 1,6, 10 and 20 years of follow-up.

- Cost-effectiveness of routine ALBC versus plain bone cement use in primary TKA.

\section{Statistical analysis}

\section{Baseline data}

Statistical analyses will be conducted under the guidance of biostatisticians at the NAR. Baseline data will be presented using descriptive statistics. $\chi^{2}$ test and independent t-test will be used to investigate differences in baseline characteristics between the two groups.

\section{Outcome data}

The power of this trial has been estimated based on noninferiority design with the non-inferiority margin set to $0.15 \%$. The non-inferiority analysis will be based on the primary outcome. Data will be analysed according to the patient's original treatment allocation in line with the intention-to-treat principles. ${ }^{77} 78$ One-year revision rate due to PJI will be estimated with the Kaplan-Meier method. Corresponding 95\% CIs will be calculated to assess possible non-inferiority of plain bone cement against fixation with ALBC. Non-inferiority is indicated if the upper limit of the 95\% CI for the absolute difference in risk of revision due to PJI does not exceed the defined inferiority margin of 0.15 . Hazard ratios will be estimated using Cox regression analyses. Subanalyses for different age groups, sex, prosthesis brands, primary diagnosis, type and dose of antibiotic used in cement, cement brand, cement mixing and delivery systems, type, dose of parallel systemic antibiotic administration, operative side (right or left knee), surgical approach, duration of surgery and type of hospital (hospital volume) will be performed. The Outcome Measures in RheumatologyOsteoarthritis Research Society International criteria for responder analyses will also be applied to calculate responder rates ${ }^{79} 80$ at 1,6 and 10 years for comparing PROM scores between the two treatment groups.

The results will be presented with $95 \%$ CI. Missing data will be investigated for any relations to the outcomes of interest (missing at random). Missing items in PROMs will be handled in accordance with guidelines for each questionnaire.

\section{Cost-effectiveness and cost-utility analysis}

The cost-effectiveness analysis will compare costs of routine ALBC and plain bone cement use in primary TKA whereas cost-utility will compare changes in the PROM scores. Markov decision analysis will be used ${ }^{81}$ (figure 2). The decision tree will represent the potential clinical course of patients in the ALBC versus plain bone cement. The periods for the cost-effectiveness and costutility analyses will be from primary TKA surgery to a 1,10 and 20 years of follow-up.

\section{Patient and public involvement}

Patients' representative has been involved in this trial project from its planning phase. Patients' representative ia a member of the Norwegian National Advisory Unit on Arthroplastyand Hip Fractures' Steering Committees. Any publications from this trial dataset will be reviewed by the trial steering committee before release. The final trial report will also be available on the NAR website. The results will be available to the public if necessary.

\section{Ethics and dissemination}

The trial has been approved by the Western Norway Regional Committees on Medical and Health Research Ethics (REK-Vest) (reference number: 2019/751/REK vest) dated: 21 June 2019. The NAR, Department of

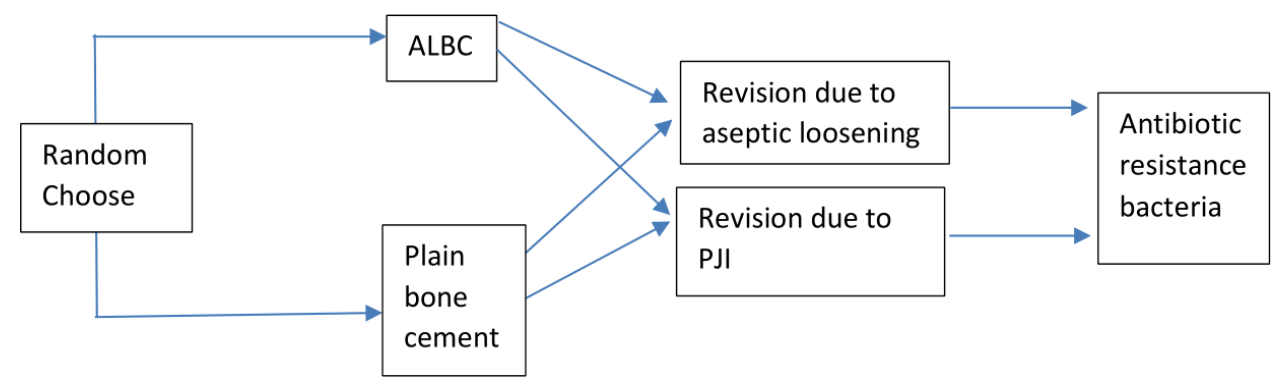

Figure 2 Schematic illustration of cost-effectiveness based on Markov model. ALBC, antibiotic-loaded bone cement. 
Orthopaedic Surgery, Haukeland University Hospital is the responsible institution for this trial. The chief investigator will coordinate dissemination of the trial data. The trial results will be reported following the Consolidated Standards of Reporting Trials Extension reporting guideline 2010 statement for non-inferiority trials. The trial results will be presented at national and international scientific conferences, and communicated to participating hospitals, patients and patient organisations, and will be published in peer-reviewed international journals. Any publications from this trial dataset will be reviewed by the trial steering committee before release. The final trial report will also be available on the NAR website.

\section{DISCUSSION}

R-RCT is a pragmatic trial that use existing registries as a platform for case identification, randomisation, clinical endpoint detection and outcome data collection. In other words, it combines a prospective randomised trial with the inclusiveness and efficiencies of a large-scale 'real-world' clinical registry. ${ }^{66}$ A standard randomised controlled trial (RCT) often has narrow inclusion criteria and low external validity, and often use surrogate endpoints to achieve adequate power within the scheduled follow-up. Observational studies have a high external validity, but are limited by selection bias and confounding. R-RCT has been successfully implemented in the Swedish Angiography and Angioplasty Register ${ }^{65}$ and has been described as a new paradigm within clinical trials. ${ }^{66}$ By randomly assigning patients in a clinical quality registry, an R-RCT combines the advantages of a standard prospective RCT with a large-scale registry study. Most importantly, R-RCT is cost-effective and time-effective, using established follow-up routines and infrastructure for data registration. ${ }^{82}$ The work load on the patient and the surgeon is minimal, and all follow-up is done electronically via webbased solutions. The NAR is a well-established register with high national coverage and good response rate. ${ }^{68}$

If, in the contrary to our hypothesis, the plain bone cement is non-inferior to the ALBC, with regards to PJI, this trial finding will go against the current practice of routine use of ALBC in primary TKA in Norway. Changing such established treatment and implementing routine use of plain bone cement may be a challenge. However, if routine use of ALBC is associated with a reduced risk of PJI and with minor impact on bacterial resistance, PROMs and costs, the well-established use of prophylactic ALBC in primary arthroplasty will be supported. Finally, the current trial will provide the framework for future pragmatic R-RCT within the Norwegian orthopaedic registries.

\footnotetext{
Author affiliations

${ }^{1}$ Faculty of Health Science, VID Specialized University, Bergen, Norway

${ }^{2}$ The Norwegian Arthroplasty Register, Department of Orthopedic Surgery, Haukeland University Hospital, Bergen, Norway

${ }^{3}$ Department of Clinical Medicine, Faculty of Medicine, University of Bergen, Bergen, Norway
}

${ }^{4}$ Department of Occupational Medicine, Haukeland University Hospital, Bergen, Norway

${ }^{5}$ Division of Orthopedic Surgery, Oslo University Hospital, Oslo, Norway ${ }^{6}$ Department of Orthopedic Surgery, St. Olavs Hospital, Trondheim, Norway ${ }^{7}$ Department of Neuromedicine and Movement Science, Faculty of Medicine and Health Science, The Norwegian University of Science and Technology, Bergen, Norway

${ }^{8}$ Department of Health Management and Health Economics, University of Oslo, Oslo, Norway

${ }^{9}$ Department of Orthopedic Surgery, Akershus Universitetssykehus HF, Oslo, Norway

${ }^{10}$ Department of Orthopaedic Surgery, Lovisenberg Diakonal Hospital, Oslo, Norway

${ }^{11}$ Department of Orthopaedic, Haugesund Hospital for Rheumatic Diseases,

Haugesund, Norway

${ }^{12}$ Department of Orthopedic Surgery, Martina Hansens Hospital, Sandvika, Norway

${ }^{13}$ Department of Orthopedic Surgery, Stavanger University Hospital, Stavanger, Norway

${ }^{14}$ Department of Medicine, Haukeland University Hospital, Bergen, Norway

${ }^{15}$ Department of Orthopedic Surgery, University Hospital of North Norway, Tromsø, Norway

${ }^{16}$ Department of Clinical Medicine, UiT-The Arctic University of Norway, Troms $\emptyset$,

Norway

${ }^{17}$ Department of Orthopedic Surgery, Namsos Hospital, Namsos, Norway

${ }^{18}$ Department of Information and Communication Technology, Western Norway

Regional Health Authority, Bergen, Norway

${ }^{19}$ Department of Information and Technology, Central Norway Regional Health Authority, Trondheim, Norway

${ }^{20}$ Coastal Hospital in Hagavik, Department of Orthopaedic Surgery, Haukeland University Hospital, Bergen, Norway

${ }^{21}$ Department of Orthopedic Surgery, Betanien Hospital, Skien, Norway

Contributors THL is a principal (chief) investigator for this trial and has taken part in initiating and planning of this trial project. Together with OF, he will play a central role in coordinating this trial project. He will also take part in data analyses, interpretation, and presentation/ dissemination of the trial results and manuscript writing. OF has taken a central role in initiating and planning this trial project. He will also be important during implementation of the trial, interpretation of the trial results, and manuscripts writing. GSD had taken part in initiating of this trial project and will participate in interpretation of the trial results and manuscript writing. AMF and SHLL are biostatisticians. They, together with THL and OF, played central role in statistical power and sample size calculation. They will give statistical support during the trial period. JEG, HD, GH, MW, and TSW had a substantial contributions to design of this trial. JEG, HD, GH, MW, TSW, ØJG, EL, AA, OEK, AKH, SH, PH, JL, $\mathrm{OSH}, \mathrm{MB}, \mathrm{RBJ}, \mathrm{SMR}, \mathrm{TB}$, and KEMA had participated in drafting and/or critically revising this trial protocol manuscript. They will participate in coordinating and providing local support to orthopedic surgeons at their hospital. They will also participate in interpretation of the trial results and manuscript writing. JPS and MW are information technology (IT) consultants. They have participated in designing electronic data registration form and the trial randomisation model for the trial together with $\mathrm{THL}$ and $\mathrm{OF}$. They will give IT-support during the trial period. $\mathrm{MW}^{*}=$ Marianne Warholm

Funding This trial is funded by the Western Norway regional health authority (Helse Vest) and grant number: HV-prosjektnr.F-10438 (912310).

Disclaimer The funding source will have no role in the collection, analysis, and interpretation of data; and in the writing of the trial report and in the decision to submit the article for publication in peer-review journals.

Competing interests None declared.

Patient consent for publication Not required.

Provenance and peer review Not commissioned; externally peer reviewed.

Supplemental material This content has been supplied by the author(s). It has not been vetted by BMJ Publishing Group Limited (BMJ) and may not have been peer-reviewed. Any opinions or recommendations discussed are solely those of the author(s) and are not endorsed by BMJ. BMJ disclaims all liability and responsibility arising from any reliance placed on the content. Where the content includes any translated material, BMJ does not warrant the accuracy and reliability of the translations (including but not limited to local regulations, clinical guidelines, terminology, drug names and drug dosages), and is not responsible for any error and/or omissions arising from translation and adaptation or otherwise. 
Open access This is an open access article distributed in accordance with the Creative Commons Attribution Non Commercial (CC BY-NC 4.0) license, which permits others to distribute, remix, adapt, build upon this work non-commercially, and license their derivative works on different terms, provided the original work is properly cited, appropriate credit is given, any changes made indicated, and the use is non-commercial. See: http://creativecommons.org/licenses/by-nc/4.0/.

ORCID iD

Tesfaye H Leta http://orcid.org/0000-0002-9596-507X

\section{REFERENCES}

1 Räsänen $\mathrm{P}$, Paavolainen $\mathrm{P}$, Sintonen $\mathrm{H}$, et al. Effectiveness of hip or knee replacement surgery in terms of quality-adjusted life years and costs. Acta Orthop 2007;78:108-15.

2 Mariconda M, Galasso O, Costa GG, et al. Quality of life and functionality after total hip arthroplasty: a long-term follow-up study. BMC Musculoskelet Disord 2011:12:222.

3 Carr AJ, Robertsson O, Graves S, et al. Knee replacement. Lancet 2012;379:1331-40.

4 Kurtz SM, Ong KL, Schmier J, et al. Future clinical and economic impact of revision total hip and knee arthroplasty. J Bone Joint Surg Am 2007;89:144-51.

5 Pabinger $\mathrm{C}$, Lothaller $\mathrm{H}$, Geissler A. Utilization rates of kneearthroplasty in OECD countries. Osteoarthritis Cartilage 2015:23:1664-73.

6 Randelli P, Evola FR, Cabitza P, et al. Prophylactic use of antibioticloaded bone cement in primary total knee replacement. Knee Surg Sports Traumatol Arthrosc 2010;18:181-6.

7 Dale H, Fenstad AM, Hallan G, et al. Increasing risk of prosthetic joint infection after total hip arthroplasty. Acta Orthop 2012;83:449-58.

8 Dyrhovden GS, Lygre SHL, Badawy M, et al. Have the causes of revision for total and Unicompartmental knee arthroplasties changed during the past two decades? Clin Orthop Relat Res 2017;475:1874-86.

9 Schroer WC, Berend KR, Lombardi AV, et al. Why are total knees failing today? etiology of total knee revision in 2010 and 2011. J Arthroplasty 2013;28:116-9.

10 Parvizi J, McKenzie JC, Cashman JP. Diagnosis of periprosthetic joint infection using synovial C-reactive protein. J Arthroplasty 2012;27:12-16.

11 Shepard J, Ward W, Milstone A, et al. Financial impact of surgical site infections on hospitals: the hospital management perspective. JAMA Surg 2013;148:907-14.

12 Chandrananth J, Rabinovich A, Karahalios A, et al. Impact of adherence to local antibiotic prophylaxis guidelines on infection outcome after total hip or knee arthroplasty. $J$ Hosp Infect 2016;93:423-7.

13 Yates AJ, American Association of Hip and Knee Surgeons EvidenceBased Medicine Committee. Postoperative prophylactic antibiotics in total joint arthroplasty. Arthroplast Today 2018;4:130-1.

14 Morris AM, Gollish J. Arthroplasty and postoperative antimicrobial prophylaxis. CMAJ 2016;188:243-4.

15 Hansen EN, Adeli B, Kenyon R, et al. Routine use of antibiotic laden bone cement for primary total knee arthroplasty: impact on infecting microbial patterns and resistance profiles. J Arthroplasty 2014;29:1123-7.

16 Engesaeter LB, Lie SA, Espehaug B, et al. Antibiotic prophylaxis in total hip arthroplasty: effects of antibiotic prophylaxis systemically and in bone cement on the revision rate of 22,170 primary hip replacements followed 0-14 years in the Norwegian arthroplasty register. Acta Orthop Scand 2003;74:644-51.

17 Qadir R, Sidhu S, Ochsner JL, et al. Risk stratified usage of antibiotic-loaded bone cement for primary total knee arthroplasty: short term infection outcomes with a standardized cement protocol. J Arthroplasty 2014;29:1622-4.

18 Mayer M, Naylor J, Harris I, et al. Evidence base and practice variation in acute care processes for knee and hip arthroplasty surgeries. PLoS One 2017;12:e0180090.

19 Sultan AA, Samuel LT, Umpierrez E, et al. Routine use of commercial antibiotic-loaded bone cement in primary total joint arthroplasty: a critical analysis of the current evidence. Ann Trans/ Med 2019;7:73.

20 Jiranek WA, Hanssen AD, Greenwald AS. Antibiotic-loaded bone cement for infection prophylaxis in total joint replacement. J Bone Joint Surg Am 2006;88:2487-500.

21 Yayac M, Rondon AJ, Tan TL, et al. The economics of antibiotic cement in total knee arthroplasty: added cost with no reduction in infection rates. J Arthroplasty 2019;34:6.
22 Parvizi J, Pawasarat IM, Azzam KA, et al. Periprosthetic joint infection: the economic impact of methicillin-resistant infections. J Arthroplasty 2010;25:103-7.

23 Cummins JS, Tomek IM, Kantor SR, et al. Cost-effectiveness of antibiotic-impregnated bone cement used in primary total hip arthroplasty. J Bone Joint Surg Am 2009;91:634-41.

24 Neut $\mathrm{D}$, van de Belt $\mathrm{H}$, Stokroos I, et al. Biomaterial-associated infection of gentamicin-loaded PMMA beads in orthopaedic revision surgery. J Antimicrob Chemother 2001;47:885-91.

25 Hendriks JGE, Neut D, van Horn JR, et al. Bacterial survival in the interfacial gap in gentamicin-loaded acrylic bone cements. $J$ Bone Joint Surg Br 2005;87:272-6.

26 Josefsson G, Kolmert L, LJCo K. Prophylaxis with systematic antibiotics versus gentamicin bone cement in total hip arthroplasty. A ten-year survey of 1,688 hips. Clin Orthop Relat Res 1993:210-4.

27 van de Belt $H$, Neut D, Schenk W, et al. Gentamicin release from polymethylmethacrylate bone cements and Staphylococcus aureus biofilm formation. Acta Orthop Scand 2000;71:625-9.

28 Hinarejos P, Guirro P, Puig-Verdie L, et al. Use of antibiotic-loaded cement in total knee arthroplasty. World J Orthop 2015;6:877-85.

29 Dunbar MJ. Antibiotic bone cements: their use in routine primary total joint arthroplasty is justified. Orthopedics 2009;32.

30 Forsythe ME, Crawford S, Sterling GJ, et al. Safeness of Simplextobramycin bone cement in patients with renal dysfunction undergoing total hip replacement. J Orthop Surg 2006;14:38-42.

31 Mounasamy V, Fulco P, Desai P, et al. The successful use of vancomycin-impregnated cement beads in a patient with vancomycin systemic toxicity: a case report with review of literature. Eur J Orthop Surg Traumatol 2013;23:299-302.

32 Dovas S, Liakopoulos V, Papatheodorou L, et al. Acute renal failure after antibiotic-impregnated bone cement treatment of an infected total knee arthroplasty. Clin Nephrol 2008;69:207-12.

33 van Raaij TM, Visser LE, Vulto AG, et al. Acute renal failure after local gentamicin treatment in an infected total knee arthroplasty. $J$ Arthroplasty 2002;17:948-50.

34 Curtis JM, Sternhagen V, Batts D, DJPTJoHP B. Acute renal failure after placement of tobramycin-impregnated bone cement in an infected total knee arthroplasty. Pharmacotherapy 2005;25:876-80.

35 Engesaeter LB, Espehaug B, Lie SA, et al. Does cement increase the risk of infection in primary total hip arthroplasty? revision rates in 56,275 cemented and uncemented primary THAs followed for $0-16$ years in the Norwegian arthroplasty register. Acta Orthop 2006;77:351-8

36 Zhou Y, Li L, Zhou Q, et al. Lack of efficacy of prophylactic application of antibiotic-loaded bone cement for prevention of infection in primary total knee arthroplasty: results of a metaanalysis. Surg Infect 2015;16:183-7.

37 Wang J, Zhu C, Cheng T, et al. A systematic review and metaanalysis of antibiotic-impregnated bone cement use in primary total hip or knee arthroplasty. PLoS One 2013;8:e82745.

38 Parvizi J, Saleh KJ, Ragland PS, et al. Efficacy of antibioticimpregnated cement in total hip replacement. Acta Orthop 2008;79:335-41

39 Hinarejos P, Guirro P, Leal J, et al. The use of erythromycin and colistin-loaded cement in total knee arthroplasty does not reduce the incidence of infection: a prospective randomized study in 3000 knees. J Bone Joint Surg Am 2013;95:769-74.

40 Gandhi R, Razak F, Pathy R, et al. Antibiotic bone cement and the incidence of deep infection after total knee arthroplasty. $J$ Arthroplasty 2009:24:1015-8.

41 Jämsen E, Huhtala H, Puolakka T, et al. Risk factors for infection after knee arthroplasty. A register-based analysis of 43,149 cases. $J$ Bone Joint Surg Am 2009;91:38-47.

42 Namba RS, Chen Y, Paxton EW, et al. Outcomes of routine use of antibiotic-loaded cement in primary total knee arthroplasty. $J$ Arthroplasty 2009;24:44-7.

43 Espehaug B, Engesaeter LB, Vollset SE, et al. Antibiotic prophylaxis in total hip arthroplasty. review of 10,905 primary cemented total hip replacements reported to the Norwegian arthroplasty register, 1987 to 1995. J Bone Joint Surg Br 1997;79:590-5.

44 Lynch M, Esser MP, Shelley P, et al. Deep infection in charnley lowfriction arthroplasty. comparison of plain and gentamicin-loaded cement. J Bone Joint Surg Br 1987;69:355-60.

45 Sanz-Ruiz P, Matas-Diez JA, Sanchez-Somolinos M, et al. Is the commercial Antibiotic-Loaded bone cement useful in prophylaxis and cost saving after knee and hip joint arthroplasty? the transatlantic paradox. J Arthroplasty 2017;32:1095-9.

46 Jameson SS, Asaad A, Diament M, et al. Antibiotic-loaded bone cement is associated with a lower risk of revision following primary cemented total knee arthroplasty: an analysis of 731,214 cases using national joint registry data. Bone Joint J 2019;101-B:1331-47. 
47 Holleyman RJ, Deehan DJ, Walker L, et al. Staphylococcal resistance profiles in deep infection following primary hip and knee arthroplasty: a study using the NJR dataset. Arch Orthop Trauma Surg 2019;139:1209-15.

48 Bistolfi A, Ferracini R, Albanese C, et al. PMMA-Based bone cements and the problem of joint arthroplasty infections: status and new perspectives. Materials 2019;12. doi:10.3390/ma12234002. [Epub ahead of print: 02 Dec 2019].

49 Sebastian S, Liu Y, Christensen R, et al. Antibiotic containing bone cement in prevention of hip and knee prosthetic joint infections: a systematic review and meta-analysis. J Orthop Translat 2020;23:53-60.

50 Rodriguez-Merchan EC. Antibiotic-loaded bone cement in primary total knee arthroplasty: does it reduce the risk of periprosthetic joint infection? Hosp Pract 2020;48:188-95.

51 Namba RS, Prentice HA, Paxton EW, et al. Commercially prepared Antibiotic-Loaded bone cement and infection risk following Cemented primary total knee arthroplasty. J Bone Joint Surg Am 2020;102:1930-8.

52 Leong JW, Cook MJ, O'Neill TW, et al. Is the use of antibiotic-loaded bone cement associated with a lower risk of revision after primary total hip arthroplasty? Bone Joint J 2020;102-B:997-1002.

53 Hoskins T, Shah JK, Patel J, et al. The cost-effectiveness of antibiotic-loaded bone cement versus plain bone cement following total and partial knee and hip arthroplasty. $J$ Orthop 2020;20:217-20.

54 Lautenschlager EP, Jacobs JJ, Marshall GW, et al. Mechanical properties of bone cements containing large doses of antibiotic powders. J Biomed Mater Res 1976;10:929-38.

55 Moran JM. Effect of gentamicin on shear and interface strengths of bone cement. Clin Orthop Relat Res 1979;\&NA:96???101-101.

56 Patrick BN, Rivey MP, Allington DR. Acute renal failure associated with vancomycin- and tobramycin-laden cement in total hip arthroplasty. Ann Pharmacother 2006;40:2037-42.

57 Bohm E, Zhu N, Gu J, et al. Does adding antibiotics to cement reduce the need for early revision in total knee arthroplasty? Clin Orthop Relat Res 2014;472:162-8.

58 Hanssen AD. Prophylactic use of antibiotic bone cement: an emerging standard--in opposition. J Arthroplasty 2004;19:73-7.

59 Bistolfi A, Massazza G, Verné E, et al. Antibiotic-loaded cement in orthopedic surgery: a review. ISRN Orthop 2011;2011:1-8.

60 Chiu FY, Lin CF, Chen CM, et al. Cefuroxime-impregnated cement at primary total knee arthroplasty in diabetes mellitus. A prospective, randomised study. J Bone Joint Surg Br 2001;83:691-5.

$612015 \mathrm{NN}-\mathrm{V}$. Usage of antibicrobial agents and occurrence of antimicrobial resistance in Norway. Tromsø/OSLo, 2016.

62 Holen Øyunn, Alberg T, Blix HS, et al. Broad-spectrum antibiotics in Norwegian hospitals. Tidsskr Nor Laegeforen 2017;137:362-6.

63 Helse- OG OMsorgsdepartment. Handlingsplan mot antibiotikaresistens I helsetjensten Oslo, 2015. Available: https:// www.regjeringen.no/contentassets/915655269bc04a47928fce91 $7 \mathrm{e} 4 \mathrm{~b} 25 \mathrm{f} 5 /$ handlingsplan-antibiotikaresistens.pdf
64 Nasjonal Kompetansetjeneste. The Norwegian arthroplasty register anual report 2020. Available: http://nrlweb.ihelse.net/eng/Rapporter/ Report2020_english.pdf

65 Fröbert O, Lagerqvist B, Olivecrona GK, et al. Thrombus aspiration during ST-segment elevation myocardial infarction. N Engl J Med 2013;369:1587-97

66 James S, Rao SV, Granger CB. Registry-based randomized clinical trials--a new clinical trial paradigm. Nat Rev Cardiol 2015;12:312-6.

67 Chan A-W, Tetzlaff JM, Altman DG, et al. Spirit 2013 statement: defining standard protocol items for clinical trials. Ann Intern Med 2013;158:200-7.

68 Nasjonal Kompetansetjeneste. The Norwegian arthroplasty register anual report 2019. Available: http://nrlweb.ihelse.net/eng/Rapporter/ Report2019_english.pdf

69 Efird J, JJljoer E. Blocked randomization with randomly selected block sizes. Int J Environ Res Public Health 2011;8:15-20.

70 Suresh K, KJJohrs S. An overview of randomization techniques: an unbiased assessment of outcome in clinical research. J Hum Reprod Sci 2011;4:8-11.

71 Karanicolas PJ, Farrokhyar F, MJCjos B. Blinding: who, what, when, why, how? Can J Surg 2010;53:345-8.

72 Sealed Envelope Ltd. Power calculator for binary outcome noninferiority trial: sealed envelope LTD, 2012. Available: https://www. sealedenvelope.com/power/binary-noninferiority/

73 Gjersvik P, Hem E, Jacobsen GW, et al. Hva bør non-inferioritystudier kalles på norsk? Tidsskriftet 2014;134:852-3.

74 O'Brien PC, Fleming TR. A multiple testing procedure for clinical trials. Biometrics 1979;35:549-56.

75 Schulz KF, Grimes DA. Multiplicity in randomised trials II: subgroup and interim analyses. Lancet 2005;365:1657-61.

76 Helsedirektoratet. Antibiotikaprofylakse ved kirurgi. Available: https:// www.helsedirektoratet.no/retningslinjer/antibiotika-i-sykehus/antibiot ikaprofylakse-ved-kirurgi/ortopedisk-kirurgi [Accessed 6 Dec 2019].

77 Detry MA, Lewis RJ. The intention-to-treat principle: how to assess the true effect of choosing a medical treatment. JAMA 2014;312:85-6.

78 McCoy CE. Understanding the intention-to-treat principle in randomized controlled trials. West J Emerg Med 2017;18:1075-8.

79 Pham T, van der Heijde D, Altman RD, et al. OMERACT-OARSI initiative: osteoarthritis research Society international set of Responder criteria for osteoarthritis clinical trials revisited. Osteoarthritis Cartilage 2004;12:389-99.

80 Escobar A, Gonzalez M, Quintana JM, et al. Patient acceptable symptom state and OMERACT-OARSI set of Responder criteria in joint replacement. Identification of cut-off values. Osteoarthritis Cartilage 2012;20:87-92.

81 Beck JR, Pauker SG, SGJMdm P. The Markov process in medical prognosis. Med Decis Making 1983;3:419-58.

82 Wachtell K, Lagerqvist B, Olivecrona GK, et al. Novel trial designs: lessons learned from thrombus aspiration during ST-segment elevation myocardial infarction in Scandinavia (taste) trial. Curr Cardiol Rep 2016;18:11. 